\title{
Der Kieler Geographentag 1969 - Modernisierungsschub, Mythos, Paradigmenwandel oder vergessene Geschichte?
}

\author{
P. Weichhart \\ Institut für Geographie und Regionalforschung, Universität Wien, Universitätsstraße 7, 1010 Wien, Austria \\ Correspondence to: P. Weichhart (peter.weichhart@univie.ac.at)
}

Received: 2 November 2015 - Accepted: 8 December 2015 - Published: 21 January 2016

Geschichte kann man als die wertende Interpretation und Deutung der Vergangenheit aus der Sicht der jeweiligen Gegenwart ansehen. So kann ein bestimmtes Ereignis der Vergangenheit aus der Perspektive der Gegenwart als besonders bedeutsam, wirkmächtig und konsequenzenreich beurteilt werden oder aber zumindest für eine Zeit in Vergessenheit geraten. Auch in der Geschichte wissenschaftlicher Disziplinen gibt es immer wieder Geschehnisse und Konstellationen, die später als Schlüsselereignisse oder Tipping Points gedeutet werden - unabhängig davon, ob das auch die Zeitgenossen so gesehen haben. Im Falle des Kieler Geographentages war die Einstufung als Schlüsselereignis sowohl zur damaligen Zeit als auch für die Jahre danach durchaus gegeben. Dieser Geographentag - beziehungsweise die dort abgehaltene Diskussionsveranstaltung, die von den geographischen Fachschaften durchgeführt wurde, sowie die ausführlichen Erörterungen und Stellungnahmen im Gefolge dieses „Events“ - wurde immer wieder als „Wende“, „Umbruch“, „Revolution“ oder Symbol eines Paradigmenwandels interpretiert. Heute, 46 Jahre danach, lässt sich natürlich die Frage stellen, ob dieses lange Zeit als höchst bedeutsam eingestufte Ereignis für die aktuelle Entwicklung der Geographie immer noch relevant ist und den gegenwärtigen Status des Faches tatsächlich nachhaltig beeinflusst hat.

Im Herbst 1969 war ich gerade im fünften Semester meines Studiums an der Universität Salzburg. Obwohl ich damals von meinen Interessenlagen her eher mit frühmittelhochdeutscher Literatur und der Theorie des Romans und weniger mit geographischen Grundsatzfragen befasst war, hatte ich aber dennoch höchst aufmerksam in den Lehrveranstaltungen von Helmut Riedl einen sehr ausführlichen Bericht über den Kieler Geographentag zur Kenntnis genommen. Da war von einer „höchst bedenklichen Entwicklung“, dem „Verlust der Einheit der Geographie“ und einer „Zerstörung der konzeptionellen Grundlagen des Faches“ die Rede. Diese Aussagen vermittelten den Eindruck, dass aus Sicht der Geographie der Untergang des Abendlandes unmittelbar bevorstehen würde. Ich fand das sehr spannend, denn zu dieser Zeit rumorte es auch in der Germanistik ziemlich heftig, und mir wurde durch diese Parallelität bewusst, dass es in den Wissenschaften nicht nur konkurrierende Auffassungen über die Deutung bestimmter Phänomene oder methodische Detailfragen gibt, sondern dass auch schwerwiegende Krisen vorkommen können, die gleichsam die Fundamente einer Disziplin betreffen. Und ich nahm mit großem Erstaunen und zunehmendem Interesse zur Kenntnis, dass Wissenschaft offensichtlich nicht der hehre Montsalvat der reinen und einzigen Wahrheit ist, sondern dass es auch innerhalb einer Disziplin konkurrierende Wahrheitspostulate gibt, die miteinander nicht in Einklang zu bringen sind und die auf jeweils unterschiedlichen und miteinander in Widerspruch stehenden axiomatischen Voraussetzungen gründen.

In der Folge begann ich, mich ernsthaft mit Wissenschaftsforschung auseinanderzusetzen und die einschlägigen Angebote der Salzburger Philosophie zu nutzen - etwa die Vorlesungen „Wissenschaftstheorie“ und „Logik“ von Paul Weingartner. Und mit den bescheidenen Werkzeugen, die ich mir dabei aneignen konnte, fing ich an, das Fach Geographie etwas genauer zu beobachten. Dabei entdeckte ich sehr rasch, wie spannend diese Disziplin ist, und versuchte, ihre Stärken und Schwächen genauer zu fassen. Dabei wandelte sich der engagierte Germanist unversehens in einen begeisterten Geographen. Für meine „Konvertierung“ und letztlich auch für die Thematik meiner Dissertation war also genau genommen der Kieler Geographentag der entscheidende Anstoß. 
Mit einigem Erstaunen musste ich in den folgenden Jahren feststellen, dass Kiel und die mit diesem Event verknüpften Implikationen für die Geographie von der weitaus überwiegenden Zahl meiner Kolleginnen und Kollegen aber gar nicht ernsthaft zur Kenntnis genommen wurden. Man machte ,business as usual“; eine ernsthafte Reflexion der Lage des Faches war für mich im Gespräch mit vielen anderen Fachvertretern nicht erkennbar. Das betraf sowohl mein fachliches Umfeld in Salzburg, dann in München und später wieder in Salzburg. In der Folge setzte ich mich intensiv mit den wichtigsten Schlüsselpublikationen rund um den Geographentag von Kiel auseinander, vor allem mit den Habilitationsschriften von Bartels (1968) und Hard (1970) sowie dem von Bartels herausgegebenen Sammelband ,Wirtschafts- und Sozialgeographie“ (Bartels, 1970), und ich versuchte auch, die Inhalte dieser Texte in meinen Lehrveranstaltungen zu vermitteln. In diesem Zusammenhang wurde mir am Beispiel eines Erlebnisses in München dann zweifelsfrei klar, dass Kiel und die Folgen für Traditionalisten in der Geographie offensichtlich doch als Trauma erlebt wurden.

Es war im Jahr 1978, also fast zehn Jahre nach Kiel, als ich in das Arbeitszimmer des damals geschäftsführenden Vorstandes des Instituts für Geographie der LMU München, Hans-Günter Gierloff-Emden, zitiert wurde. Mit barscher Stimme und ohne Begrüßungsformeln herrschte er mich an: „Mir ist zu Ohren gekommen, dass Sie in Ihren Lehrveranstaltungen Autoren wie Gerhard Hard und Dietrich Bartels zitieren. Ich wünsche, dass diese Namen an meinem Institut nicht einmal erwähnt werden. Habe ich mich deutlich genug ausgedrückt?" Meine Reaktion, dass Wissenschaft und ihre Lehre frei seien, wischte er mit einer mich entlassenden Handbewegung beiseite.

Als „Ehrentiroler“1 hat mich dieses Erlebnis natürlich nur darin bestärkt, mich weiter mit den Schriften der „Aufmüpfigen“ sowie den Reaktionen von Vertretern des klassischen Mainstreams auseinanderzusetzen. Dabei fand ich auch Textstellen von durchaus hohem Unterhaltungswert, etwa das Streitgespräch zwischen Dörrenhaus (1971) und Hard (1971). Je länger ich mich aber mit dem von den Revolutionären propagierten Spatial Approach befasste, desto deutlicher wurde mir klar, dass dieser Ansatz zwar intellektuell höchst anregend ist und für eine beachtliche Zahl geographischer Fragestellungen hervorragende Lösungsansätze und methodische Werkzeuge bereitstellt, sich für viele andere Problemlagen aber ganz und gar nicht eignet. Und mir wurde zunehmend bewusst, dass der zentrale Kern des Programms von Dietrich Bartels, nämlich die ,wissenschaftstheoretische Grundlegung einer Geographie des Menschen“ mit diesem Absolutheitsanspruch gar nicht einlösbar sein kann. Die Beschäftigung mit der verhaltenswissenschaftli-

\footnotetext{
${ }^{1}$ Helmut Heuberger, mein damaliger Chef und väterlicher Freund in München und später in Salzburg, hatte mehrfach angemerkt, dass ich ob meiner Sturheit und Hartnäckigkeit jederzeit als Tiroler durchgehen könne.
}

chen Geographie und vor allem mit Handlungstheorien führte mich $\mathrm{zu}$ anderen Wissenschaftstheorien und alternativen Möglichkeiten von „Grundlegungen“ einer Geographie des Menschen (Weichhart, 1988). Und damit landete ich schließlich konsequenterweise auch bei der Paradigmenforschung (vgl. Schurz, 2012).

Obwohl ich also gar nicht dabei war, den fachgeschichtlichen und gesellschaftlichen Kontext sowie den relevanten Quellen erst nach und nach Beachtung schenkte und im Studium noch voll in die klassische Einheitsgeographie sozialisiert worden war, hat der Kieler Geographentag meine persönliche fachliche Entwicklung zweifellos grundlegend und nachhaltig beeinflusst. Aus diesem Einzelfall (?) lassen sich aber natürlich keinerlei Folgerungen ableiten, die man für das Gesamtfach verallgemeinern könnte. Um die Relevanz dieses Ereignisses für die Geographie besser und objektivierbarer abschätzen zu können, möchte ich im Folgenden zunächst kurz auf eine zentrale Quelle, den ,Tagungsbericht“ (Meckelein und Borcherdt, 1970) des Kieler Geographentages, eingehen.

Auf den ersten Blick handelt es sich durchaus um einen typischen Tagungsband ohne spektakuläre Besonderheiten. Im Vorwort bemerken die Herausgeber, dass die Hauptthemen der Sitzungstage ,... wieder fast die ganze Spannweite der Geographie auf[zeigen]" (Meckelein und Borcherdt, 1970, Vorwort). Jedoch sei auf eine Besonderheit hingewiesen: „Zum ersten Mal wurden in der Öffentlichkeit eines Geographenkongresses die Probleme der Studien- und Berufsproblematik sowie der Didaktik gemeinsam von Hochschullehrern, Schul- und Berufsgeographen sowie Studierenden diskutiert. In den zum Teil fast leidenschaftlichen Auseinandersetzungen darüber offenbarte sich eine gewisse Unsicherheit, vor allem unter den jüngeren Teilnehmern, gegenüber den bisherigen Grundlagen, Methoden und Zielen der geographischen Wissenschaft. Nun hat eine solche Phase wohl jeder Geograph einmal durchgemacht. Aber es zeigte sich in Kiel doch ein so weit verbreitetes Interesse an den allgemeinen methodischen Fragen, dass diese Diskussion in Zukunft wird weitergeführt werden müssen“ (ebd.; Hervorhebungen P. Weichhart). Trotz des perfekten Understatements (zum Teil fast leidenschaftlich ...gewisse Unsicherheit) in den Formulierungen wird für den aufmerksamen Leser gleichsam zwischen Zeilen aber doch klar, dass hier wohl etwas Auffälliges angesprochen wird.

In der „Ansprache des scheidenden 1. Vorsitzenden des Zentralverbandes der Deutschen Geographen“"von Wolfgang Meckelein (Meckelein und Borcherdt, 1970:33-37) zum Abschluss des Geographentages werden die Vorkommnisse dann etwas präziser formuliert. Die Studien- und Berufsproblematik hätte eine leidenschaftliche Diskussion ,,entfesselt", die deutlich machte, dass ,... eine bedrückende Unsicherheit über das besteht, was Geographie eigentlich ist, aber auch darüber, was Geographie heute sein soll oder sein kann. Am extremsten ist das wohl von Seiten der Studenten ausgedrückt worden, die gerade die Grundlagen eines guten 
Teils der Methodik der Geographie und ihre theoretische Basis in Frage gestellt haben" (S. 34). Eingeschoben in diese Ansprache ist ein knappes Statement des damaligen Studenten J. Neupert (S. 34-35). Die von den Hochschullehrern vorgeschobene ungenügende Vorbereitungszeit zur Beantwortung studentischer Anfragen erscheint ihm aus studentischer Sicht doch „erschütternd“, denn jeder Dozent der Geographie sollte doch in der Lage sein, ,... die Grundlagen der geographischen Wissenschaft jederzeit argumentativ zu vertreten“. Ein starkes Argument, das wohl geschmerzt haben muss zumindest jene Zuhörer, welche die Situation ernsthaft reflektiert hatten.

Wolfgang Meckelein (S. 35) drückt es in der Folge sehr klar und unmissverständlich aus: „Es erscheint notwendig und richtig, dass wir uns in der Geographie wieder mehr mit methodischen Grundfragen beschäftigen. Nicht nur aus dem zufälligen Interesse eines einzelnen heraus, sondern auch um die Grundfragen unseres Faches möglichst transparent an jeden unserer Studierenden herantragen zu können, muss darüber diskutiert werden. So wird jeder für sich selbst verdeutlichen können und müssen, was wir mit Erschrecken als Mangel auf eben diesem Kongress haben feststellen können: die Frage nach dem Sinn der geographischen Wissenschaft überhaupt!“ Besonders deutlich formuliert dies auch Peter Schöller in seiner Ansprache als neuer 1. Vorsitzender des Zentralverbandes Deutscher Geographen (Meckelein und Borcherdt, 1970:37-38): ,So würde ich es für falsch und gefährlich halten, wenn einige von uns diesen Geographentag mit dem Gedanken verließen: Wir sind noch einmal davongekommen. Das wäre gefährlich, weil es nicht stimmt. Wir sind nicht davongekommen, und wir werden nicht davonkommen, immer wieder und immer neu die Existenzfragen unseres Faches zu stellen, unserer Forschung, unserer Ausbildung und unserer Praxis“" (S. 38).

Diese wenigen Zitate können wohl verdeutlichen, dass Kiel als eine Art „Erweckungserlebnis“ für zumindest einige der Teilnehmer zu interpretieren ist, durch das sie auf die Notwendigkeit und Dringlichkeit kritischer Selbstreflexionen verwiesen wurden. Ich möchte dies als wichtiges Element eines Modernisierungsschubes ansehen, der für unser Fach durch die Ereignisse von Kiel angestoßen wurde.

Die im Tagungsband als Abschnitt III folgenden Abhandlungen bieten bis S. 173 keinerlei Auffälligkeiten, die sich von einem „Standard-Geographentag“ unterscheiden würden. Ernsthaft zur Sache geht es dann mit dem Abschnitt 6 (,Der Geograph - Ausbildung und Beruf“). Aus den Worten zur Eröffnung der Sitzung wird deutlich, dass es im Vorfeld der Veranstaltung offensichtlich bereits Absprachen, Übereinkünfte und „diplomatische“ Aktivitäten gegeben haben muss. Es wurde bei der Vorbereitung wohl so etwas wie ein professionelles Krisenmanagement betrieben, bei dem das Establishment der Hochschulgeographen durchaus versucht hat, die ,aufmüpfigen“ Studierenden schon vorweg etwas zu zügeln und die Veranstaltung in geordneten Bahnen ablaufen zu lassen (siehe unten). Peter Schöller (S. 175) ersucht in seinen Eröffnungsworten darum, die Veranstaltung ,emotionslos“ abzuführen: „Bei aller Konsequenz der Argumente sollte nie die Achtung und der Respekt vor der Meinung des anderen verletzt werden." Es muss auch so etwas wie eine Vereinbarung gegeben haben, nicht vom vorbereiteten (und bei einer Besprechung in Köln gleichsam vorab ,abgesegneten") Text abzuweichen.

Heinz W. Friese (Meckelein und Borcherdt, 1970:177182) referiert dann über ,Thesen zur Ausbildung der künftigen Geographielehrer“, die auf die „Inkongruenz von Schulgeographie und Hochschulgeographie" (S. 179) verweisen. Er stellt fest, dass die immer wieder beschworene Einheit der Geographie, sofern sie „... überhaupt jemals bestanden hat, ... in zunehmendem Maße Fiktion ..." geworden sei (S. 178). Darauf folgen ,Thesen zur Ausbildung des Diplomgeographen“ von Karl Ganser (Meckelein und Borcherdt, 1970:183-190). Seine sehr plausiblen Thesen, auf die an dieser Stelle nicht näher eingegangen werden soll ${ }^{2}$, stellt er dem ,... gegenwärtig noch weit verbreiteten Selbstverständnis der Geographie ... “ gegenüber, was ,einige Diskrepanzen" aufzeige (S. 187). Gesellschaftspolitisch bedeutsame Fragen würden in der aktuellen Forschungsarbeit ,eine untergeordnete Rolle" spielen (ebd.). Besonders deutlich wird herausgestellt: „Das zähe Festhalten am Landschaftskonzept, gedacht als meiner Ansicht nach unlösbare Universalaufgabe, alle raumbezogenen Erscheinungen zu erfassen, gedacht als wohl immer weniger wirksame Zauberformel der Eigenständigkeit unseres Faches, behindert die methodische Weiterentwicklung in Richtung quantitativ modellhafter Arbeitsweisen und aspektbezogener Spezialisierung" (S. 187). Und daher sei es auch ein Anachronismus, ,... einen Diplomgeographen als physischen und als sozialwissenschaftlichen Geographen ausbilden zu wollen. Beide Studiengänge müssen getrennt werden“" (S. 189).

Anschließend folgt die berühmt-berüchtigte „Bestandsaufnahme zur Situation der deutschen Schul- und Hochschulgeographie“ (Meckelein und Borcherdt, 1970:191-207), die von einer Redaktionsgruppe der Fachschaften vorgelegt wurde. Aus heutiger Sicht handelt es sich um einen geradezu gemäßigt formulierten und fast harmlosen Text, der sich allerdings aus damaliger Sicht durchaus als ,revolutionäres Manifest" interpretieren lässt. Das Image des Schulfaches Geographie wird vor dem Hintergrund von Umfragen in aller Deutlichkeit als überaus schlecht identifiziert. Aus der ,aufgezeigten Misere“ werden dann zwei Thesen abgeleitet:

1. „Die Geographie entzieht sich ihren Aufgaben und ihrer Verantwortung innerhalb der Gesellschaft."

2. „Darüber hinaus kann Geographie, soweit sie sich als Landschafts- und Länderkunde begreift, nicht einmal wissenschaftlichen Ansprüchen gerecht werden." (S. 193)

\footnotetext{
${ }^{2}$ Vgl. dazu Wardenga et al. (2011:55-61).
} 
Ausdrücklich wird betont, dass Wissenschaft nicht wertfrei arbeiten könne und die Verpflichtung habe, kritisches Wissen zu liefern (S. 194). Der Erdkundeunterricht müsse sich funktional innerhalb des Bildungsprozesses ansiedeln und kritische Analysen bereitstellen. Die Vorrangstellung der Länderkunde in den Lehrplänen sei aufzugeben.

Sehr deutlich wird herausgestellt, dass das Landschaftskonzept die zentrale Begründung für die Vorstellung einer eigenständigen und einheitlichen ,Gesamtwissenschaft“ darstellt. Und mit dem Tabu des Landschaftskonzepts solle im Folgenden gebrochen werden, weil es die wissenschaftliche Arbeit in der Geographie behindere. Denn: Ein ständiges Fortschreiten im Erkenntnisprozess bedarf der Theorie (S. 196). Diese ausdrückliche Betonung der Notwendigkeit von Theorie halte ich für den zweiten bedeutsamen Aspekt des Entwicklungsschubs, der durch den Geographentag in Kiel in Gang gesetzt wurde. „Landschaft als einheitsstiftenden Objekt ist axiomatische Voraussetzung und definiertes Ergebnis geographischer Arbeit. Um diesen Zirkel nicht einzugestehen, produziert die Geographie überall dort diffuse Leerformeln, wo die Legitimation als Wissenschaft eingelöst werden muss“ (S. 197). „Wir wollen ... aus der Sackgasse der Landschafts- und Länderkunde ausbrechen, um Raum zu schaffen für eine Diskussion über neue theoretische Ansätze. So, und nur so kann die Überlebenschance der Geographie gewahrt bleiben“ (S. 197). „Geographie als Landschaftsund Länderkunde ist Pseudowissenschaft" (S. 199).

Abschließend wird eine Reihe von Forderungen für eine zeitgemäße Ausbildung gestellt. Davon sei nur das unter Punkt 9 formulierte Postulat zitiert: „Die Trennung von Physischer Geographie und Anthropogeographie muss vollzogen werden. Die Gemeinsamkeit der Probleme ist nur künstlich aufrechtzuerhalten“ (S. 201). Insgesamt stellt dieser Text eine kritische und hervorragend formulierte Diagnose des damaligen Status der Geographie dar, die aus heutiger Sicht von den meisten Vertretern des Faches geteilt werden muss wenngleich dies von nicht wenigen auch bedauert wird.

Die folgende Diskussion zur Sitzung fällt bei den meisten Wortmeldungen, was Klarheit und Schlüssigkeit der Argumentation betrifft, gegenüber der ,Bestandsaufnahme“ deutlich ab. Da wird von „Ideologisierung“, „übergreifendem Denken“ (S. 210), dem ,Interesse der Öffentlichkeit an Länderstudien“ (S. 211), der ,synthetischen Leistung“ der Länderkunde (S. 213), vom „Verständnis der Wirkungsgefüge“ (S. 214), von ,zu komplexen und zu komplizierten Systemen“ (S. 216) oder von den Schwächen eines „Ad-hocDisputs“ (S. 217) gesprochen. Die Formulierungen der Studierenden zum Thema Länderkunde seien ,,schlicht und einfach unwissenschaftlich“ (S. 219), die Studenten hätten ,,aus dem Zusammenhang gerissene Zitate“ verwendet (S. 230), sie hätten in ,krampfhaftem Ton“ und mit ,völligem Mangel an Humor" gesprochen (S. 230). Ernsthafte Sachargumente sind bei den Wortmeldungen des „Establishments“ eher selten.
Der restliche Teil des „Tagungsberichts“ bietet wieder „business as usual“.

Geistesgeschichtlich gesehen kann man aus der Durchsicht dieser zentralen Quelle also durchaus ein wirklich sensationelles Ereignis oder eine dramatische Revolution konstatieren, bei der kein Stein auf dem anderen geblieben ist. Die Aussagen und Forderungen in der „Bestandsaufnahme“ der Studierenden sind mit den konzeptionellen Grundannahmen der klassischen Geographie absolut nicht vereinbar. Kiel lässt sich somit zweifellos als Symbol für einen Paradigmenwandel im Sinne von Kuhn (1962) interpretieren, auch wenn es keine unmittelbaren Folgewirkungen für die Handlungspraxis des Faches gab. Die Mainstream-Geographie hat sich nach Kiel keineswegs schlagartig und grundlegend verändert, sondern verblieb noch längere Zeit im gewohnten Fahrwasser. Zur genaueren Beurteilung der Situation müsste man natürlich auch noch andere Quellen heranziehen, etwa die Geografiker-Texte und etliche kritische Veröffentlichungen, die schon vor 1969 publiziert wurden, denn die Bühne für die Revolution von Kiel war damals längst vorbereitet. Es gab eine ganze Reihe von Autoren, die bereits vor Kiel an der Sinnhaftigkeit der Einheitsgeographie gezweifelt (etwa Alfred Rühl schon 1933) oder das Landschaftskonzept in Frage gestellt und als Hypostasierung entlarvt hatten (etwa Walter Gerling, z.B. 1965). Diese beiden Fragen hängen ja unmittelbar miteinander zusammen, denn das Landschaftskonzept war die (einzige) theoretische Begründung für die Notwendigkeit der Einheit des Faches. In mehreren Publikationen war schon lange vor Kiel von einer „Malaise“ der Geographie die Rede. Jedenfalls entspricht die hier vorfindbare wissenschaftsgeschichtliche Situation exakt jenem Modell, das in der evolutionstheoretischen Konzeption von Thomas S. Kuhn einen Paradigmenwandel beschreibt.

Allerdings folgte die weitere Entwicklung des Faches keineswegs der „radikalen Lesart“ Kuhns, der davon ausging, dass ein ,altes“ Paradigma endgültig verdrängt und das neue (also das in Kiel propagierte raumwissenschaftliche Paradigma) mit dem Erreichen der ,normalwissenschaftlichen Phase“ für längere Zeit dominant bleibt (vgl. Weichhart, 2012; Kornmesser und Schurz, 2014). Das Paradigma der klassischen Landschafts- und Länderkunde blieb noch längere Zeit aktuell, erreichte immer wieder neue Phasen eines ,Konjunkturaufschwunges" und ist auch heute noch als Reliktform eines Paradigmas (zum Teil als unreflektierte „Kryptotheorie") erkennbar. Der quantitativ ausgerichtete und neopositivistisch orientierte Spatial Approach hat auch keineswegs eine hegemoniale Dominanz erlangt, sondern wurde rasch durch andere und miteinander konkurrierende Paradigmen ergänzt. Der gegenwärtige Status des Faches ist durch eine multiparadigmatische Struktur gekennzeichnet, bei der mehrere Paradigmen koexistieren. Ist die Revolution von Kiel also gescheitert? Nein, gewiss nicht. Die weitere Entwicklungsgeschichte des Faches hat damit vielmehr genau jene Struktur angenommen, die in der ,Post-Kuhn “schen“ Paradigmendiskussion beschrieben wird (vgl. Schurz, 2014). 
Welche Bedeutung hat der Kieler Geographentag für den heutigen Status der Geographie? Wird er heute noch als wirkmächtiges Ereignis interpretiert, hatte er für die persönliche fachliche Entwicklung gegenwärtig aktiver Fachkollegen und vor allem für die jüngere Generation irgendeine Relevanz? Um Antworten auf solche Fragen finden zu können, habe ich versucht, im Rahmen einer kleinen Umfrage ${ }^{3}$ die „Stimmungslage“ zu diesem Thema zu erheben.

Die Reaktionen auf diese persönlich formulierte Anfrage waren für mich einigermaßen überraschend. Nur etwas mehr als zehn Prozent der Befragten haben auf meine EMail reagiert. Das mag mit dem Zeitpunkt der Erhebung kurz vor Semesterbeginn und dem damit verbundenen hohen Arbeitsanfall zu tun haben. Es kann aber auch bedeuten, dass „Kiel“ für einen großen Teil der Angeschriebenen kein ausgesprochenes Reizwort ist. Diese Vermutung wird dadurch bestärkt, dass bei zwei der Respondenten (durchaus ,höhere Semester") aus der Antwort deutlich ersichtlich wurde, dass sie mit dem Stimulus „Geographentag Kiel 1969“ nicht das Geringste anfangen konnten. Es könnte also durchaus sein, dass „Kiel“ für einen nicht geringen Teil der aktuell tätigen Hochschulgeographen so etwas wie ,vergessene Geschichte“ darstellt. Ein weiteres Indiz dafür ist der Hinweis einer (jüngeren) Geographin, dass sie im Verlaufe ihres Studiums nie etwas über dieses Ereignis gehört hatte. Einer ihrer akademischen Lehrer hatte ihr aber im Rahmen einer Tagung darüber erzählt und ihr dabei sehr eindrücklich vermittelt, dass Kiel für seine Generation ein ,einschneidendes und revolutionäres Ereignis gewesen ist, was unter anderem auch dafür gesorgt habe, dass Human- und Physiogeographinnen in die wechselseitige Sprachlosigkeit (und auch teilweise Geringschätzung) gefallen sind“. Ob der Kieler Geographentag für den gegenwärtigen Status der Geographie bedeutsam sei, könne sie nicht beurteilen, weil sie einfach zu uninformiert über die Geschehnisse und Folgen sei.

Umgekehrt betonen zwei (jüngere) Befragte, dass sie im Rahmen ihrer Ausbildung sehr gut über den Kieler Geographentag und seine Folgen informiert worden seien, und belegen mit ihren Aussagen, dass sie die disziplingeschichtliche Bedeutung dieses Ereignisses durchaus kompetent beurteilen können. Daraus ergibt sich für mich die Folgerung, dass es im Rahmen der Studienpläne unbedingt erforderlich ist, Lehrveranstaltungen vorzusehen, die sich mit der Paradig-

\footnotetext{
${ }^{3}$ Es handelte sich hier um eine Befragung per E-Mail, die ich in Form eines persönlichen Schreibens an etwa 75 Kolleginnen und Kollegen an mehreren Geographieinstituten gerichtet habe. Die Umfrage entsprach nicht den strengen methodischen Anforderungen eines solchen Erhebungsinstrumentes, sondern war ausdrücklich als persönliche Anfrage formuliert. Die Angeschriebenen wurden gebeten, kurz auf die Bedeutung des Kieler Geographentages für die eigene fachliche Entwicklung einzugehen und aus subjektiver Sicht abzuschätzen, ob und in welcher Form die Ereignisse von Kiel aus heutiger Sicht für den gegenwärtigen Zustand des Faches relevant seien.
}

menentwicklung des Faches und seiner „Dogmengeschichte“ ernsthaft auseinandersetzen.

Mehrere Teilnehmer betonten, dass durch das Ereignis von Kiel eine ,theoretische Wende" herbeigeführt wurde, welche für die weitere Fachentwicklung überaus bedeutsam gewesen sei und somit auch den aktuellen Status des Faches bestimmen würde. Dies sei auch dafür verantwortlich, dass sich GeographInnen heute problemlos mit anderen Sozialwissenschaften austauschen können.

Besonders interessant scheint mir die Reaktion eines Vertreters der Physiogeographie. Auch er hat den Kieler Geographentag durch Berichte seiner akademischen Lehrer gleichsam aus zweiter Hand kennengelernt. Nach seiner Einschätzung war dieses Ereignis Ausdruck einer Zeit, ,in der man bereit war, alles zu hinterfragen, was Vor- und Nachteile hat“: „Einerseits hat man es im Nachgang verpasst, grundsätzliche Fragen und vielleicht auch Lebenslügen der Geographie zu klären, andererseits wäre die konsequente Klärung vielleicht eher eine Selbstzerfleischung geworden, die die Bearbeitung von realen Problemen verzögert/verhindert hätte“. Im Gefolge des Ereignisses sei vielleicht eine Atmosphäre entstanden, in der man sich nicht mehr traut, grundsätzliche Fragen aufzuwerfen. Die Rückwirkungen auf seine persönliche fachliche Entwicklung fasst er folgendermaßen zusammen: „Mir ist u.a. durch die Berichte der vielen Diskussionen über Sinnfragen der Geographie die Lust an Sozialgeographie vergangen und ich habe mich dann lieber den Naturwissenschaften zugewandt, wo man weniger über sich selbst spricht." Auf die Frage, ob die Ereignisse von Kiel und die Folgediskussionen für den gegenwärtigen Status der Geographie bedeutsam seien, reagierte er wie folgt: „Ich glaube schon. Und zwar insofern, als dass die Geographen nun lieber nebenher arbeiten und sich in Ruhe lassen als ihre Beziehung zueinander zu klären. Ob ich das gut oder schlecht finde, hängt von meiner Stimmung ab".

Einer der Befragten interpretiert Kiel vor allem als Generationenkonflikt. Die Berichte in Lehrveranstaltungen junger Assistenten waren aus seiner Sicht ,persönlich gefärbt" und stark zu einem personalisierten Bild des ,heroischen Aufbegehrens der Jungen gegenüber einem alten und männlichen Establishment" komprimiert. Zwischen den Zeilen ist zu lesen, dass seine Sympathie damals wohl eher auf der Seite des Establishments lag.

Eine besonders ausführliche und informative Stellungnahme erhielt ich von Hans-Heinrich Blotevogel ${ }^{4}$. Auch er war in Kiel nicht persönlich anwesend, war aber indirekt nahe am Geschehen, weil sein akademischer Lehrer Peter Schöller als Vorsitzender des Zentralverbandes verantwortlich für das Programm des Geographentages war. „Schöller vertrat im Verband eine liberale Linie (ähnlich wie Hartke, Wirth, Sandner und Lenz), also eine Minderheitenposition gegenüber den mehrheitlich sehr konservativ eingestellten Fach-

\footnotetext{
${ }^{4}$ Er hat mich ausdrücklich autorisiert, seine Aussagen personalisiert zu zitieren.
} 
kollegen (wie z.B. Meckelein und Otremba). Schöller war bewusst, dass sich vor dem Geographentag in den Fachschaften etwas zusammenbraute. Die Fachschaften hatten ein Vorbereitungstreffen (ich glaube es war in Köln), das prinzipiell öffentlich war. Schöller fuhr dort einfach hin, um zu erfahren, was dort vor sich ging. Es war nämlich zu befürchten, dass in den Fachschaften die radikalen Vertreter die Oberhand gewannen mit der Perspektive, dass der ganze Geographentag in Kiel gesprengt worden wäre. Schöller erreichte dann, dass das Anliegen der Fachschaften in einer offiziell angesetzten Diskussionssitzung vorgebracht und diskutiert werden konnte. Er hat dafür von den konservativen Kollegen ziemlich Kritik einstecken müssen. Andererseits erreichte er, dass sich unter den Studierenden die gemäßigten Reformer durchsetzten.“

„Für mich persönlich bedeutete der Geographentag den Aufbruch zu einer grundlegenden szientifischen Modernisierung. ... Mein Lehrer Schöller war als halber Historiker eher ein Hermeneutiker; er verstand sich aber sehr gut mit D. Bartels und beide waren sich darin einig, dass es in der Humangeographie eine analytisch-nomologische Richtung und eine historisch-hermeneutische Richtung geben solle. ... Übrigens waren sich beide auch darin einig, dass die Gesamtgeographie durch die neueren Entwicklungen zur Chimäre geworden war (Sie hätten das aber nie öffentlich geäuBert).“

Zweifellos habe der Kieler Geographentag nach Meinung Blotevogels ,...einen längst überfälligen frischen Wind in die deutschsprachige Geographie gebracht. Dabei wirkte der Geographentag aber nicht als Ursache, sondern eher als Katalysator einer Entwicklung, die längst im Gange war und international auch schon viel weiter vorangeschritten war. Ich bin sicher, dass die szientifische Modernisierung auch ohne den Kieler Geographentag die deutschsprachige Geographie erreicht hätte, aber so wirkte der Geographentag als Modernisierungs-Push." Langfristige Wirkungen auf die Fachentwicklung sieht er in dreifacher Hinsicht. Die Geographie erreichte in der Folge ein höheres methodologisches Reflexionsniveau. „Damit zusammen hing die Einsicht, dass die Geographie nicht nur Fakten sammeln und beschreiben solle, sondern empirisch gehaltvolle Theorien aufstellen möge. Überhaupt wurde erst jetzt die Bedeutung von Theorien erkannt (auch wenn der dumpfe Empirismus immer noch nicht ausgestorben ist). Drittens schließlich wurde die Rolle der Wissenschaft in der Gesellschaft reflektiert und damit die Frage nach der gesellschaftlichen Relevanz von Wissenschaft gestellt."

Zusammenfassend lässt sich also festhalten, dass der Kieler Geographentag von 1969 als Symbol für einen Paradigmenwandel im Fach Geographie verstanden werden muss. „Symbol“ deshalb, weil ein Pardigmenwandel im Verständnis Kuhns (und auch im heutigen Paradigmenverständnis) als ein längerfristiger Prozess anzusehen ist, der nicht auf ein Einzelereignis reduziert werden kann. Die Veranstaltung ist damit auch als Mythos wirksam. Denn allein die Nennung dieses Ereignisses evoziert beim (informierten) Gesprächspartner die Vorstellung eines komplexen Gefüges der wissenschaftsgeschichtlichen, sozialen, methodischen und methodologischen Zusammenhänge, die für einen Paradigmenwandel charakteristisch sind, ohne dass der Sprecher diese $\mathrm{Zu}$ sammenhänge ausführlich darstellen und begründen müsste. ${ }^{5}$ Der Kieler Geographentag ist zweifellos auch als Auslöser eines Modernisierungsschubs für das Fach Geographie zu sehen. Dies wirkte sich besonders darauf aus, dass die „Theorien-Phobie“ der klassischen Geographie heute weitgehend überwunden ist. Die Auswirkungen auf das Fach und auch die Karrieren von Geographen und Geographinnen sind auch aus heutiger Sicht als sehr erheblich einzuschätzen. Gleichzeitig ist Kiel 1969 für zahlreiche Fachvertreter (nicht nur für jüngere) aber auch vergessene Geschichte.

Das „oder“ im Titel dieses Beitrages ist also durch ein „und" zu ersetzen.

Danksagung. Ich bedanke mich herzlich bei all jenen Kolleginnen und Kollegen, die meine „Blitzumfrage“ beantwortet haben.

Edited by: B. Korf

Reviewed by: one anonymous referee

\section{Literatur}

Bartels, D.: Zur wissenschaftstheoretischen Grundlegung einer Geographie des Menschen, Erdkundliches Wissen - Geographische Zeitschrift, Steiner Verlag, Wiesbaden, Beiheft Nr. 19, 1968.

Bartels, D. (Hrsg.): Wirtschafts- und Sozialgeographie, Neue Wissenschaftliche Bibliothek, 35 - Wirtschaftswissenschaften, Kiepenheuer \& Witsch, Köln, 1970.

Boesch, E. E.: Symbolic Action Theory and Cultural Psychology, Recent Research in Psychology, Springer Verlag, Berlin, 1991.

Dörrenhaus, F.: Geographie ohne Landschaft? Zu einem Aufsatz von Gerhard Hard, Geographische Zeitschrift, 59, 101-116, 1971.

Gerling, W.: Der Landschaftsbegriff in der Geographie: Kritik einer Methode, Stahel, Würzburg, 1965.

Hard, G.: Die „Landschaft“ der Sprache und die „Landschaft“ der Geographen. Semantische und forschungslogische Studien zu einigen zentralen Denkfiguren in der deutschen geographischen Literatur, Geographisches Institut Universität Bonn, Bonn, Colloquium Geographicum, Bd. 11, 1970.

Hard, G.: Ärger mit Kurven, Geographische Zeitschrift, 59, 277289, 1971.

Kornmesser, St. und Schurz, G. (Hrsg.): Einleitung und Übersicht, in: Die multiparadigmatische Struktur der Wissenschaften, VS Verlag für Sozialwissenschaften, Springer Fachmedien Wiesbaden, 11-46, 2014.

Kuhn, Th. S.: The Structure of Scientific Revolutions, International Encyclopedia of Unified Science, The University of Chicago Press, Chicago, 1962.

5 ,A myth is a system of explanation and justification for which no rational proof or deduction is or can be given“ (Boesch, 1991:123). 
Meckelein, W. und Borcherdt, Ch. (Hrsg.): Tagungsbericht und wissenschaftliche Abhandlungen, Deutscher Geographentag, 2126 Juli 1969, Kiel, Verhandlungen des deutschen Geographentages, Steiner Verlag, Wiesbaden, Band 37, 1970.

Rühl, A.: Einführung in die allgemeine Wirtschaftsgeographie, A. W. Sijthoff, Leiden, 1933.

Schurz, G.: Koexistenz rivalisierender Paradigmen: Ergebnisse und Folgen eines Salzburger Spezialforschungsbereichs, in: Anthologie zur Sozialgeographie, Herausgeber: Weixlbaumer, N., Abhandlungen zur Geographie und Regionalforschung, Universität Wien, Band 16, 125-137, 2012.

Schurz, G.: Koexistenz und Komplementarität rivalisierender Paradigmen: Analyse, Diagnose und kulturwissenschaftliches Fallbeispiel, in: Die multiparadigmatische Struktur der Wissenschaften, Herausgeber: Kornmesser, St. und Schurz, G., Springer Fachmedien, Wiesbaden, 47-62, 2014.
Wardenga, U., Henniges, N., Brogiato, H. P., und Schelhaas, B.: Der Verband deutscher Berufsgeographen 1950-1979, Eine sozialgeschichtliche Studie zur Frühphase des DVAG, Forum IfL, Leibniz-Institut für Länderkunde, Leipzig, Heft 16, 2011.

Weichhart, P.: Geography as a "Multi-Paradigm-Game" - a Pluralistic Discipline in a Pluralistic Post-Industrial Society, in: The Role of Geography in a Post-Industrial Society, Herausgeber: Windhorst, H.-W., Vechtaer Arbeiten zur Geographie und Regionalwissenschaft, Band 5, 49-54, 1988.

Weichhart, P.: Wie „funktioniert“ ein Paradigma?, in: Geographie für eine Welt im Wandel, Herausgeber: H. Fassmann, H. und Glade, T., 57. Deutscher Geographentag 2009, Wien, V\&R unipress, Göttingen, 53-64, 2012. 